\title{
Antifungal Drug Resistance - Concerns for Veterinarians
}

\author{
Bharat B. Bhanderi, Mahendra Mohan Yadav and Ashish Roy \\ Department of Veterinary Microbiology \\ College of Veterinary Science and Animal Husbandry, Anand Agricultural University, \\ Anand, (Gujarat) - 388001
}

\begin{abstract}
In the 1990s, there were increased incidences of fungal infectious diseases in human population which might be due to increase in immunosuppressive diseases. But the major concern was increase in prevalence of resistance to antifungal drugs which were reported both in the fungal isolates of human beings and that of animal origin. In both animals and human beings, resistance to antimicrobial agents has important implications for morbidity, mortality and health care costs, because resistant strains are responsible for bulk of infection in animals and human beings, and large number of antimicrobial classes offers more diverse range of resistance mechanisms to study and resistance determinants move into standard well-characterized strains that facilitates the detailed study of molecular mechanisms of resistance in microorganisms. Studies on resistance to antifungal agents has been lagging behind that of antibacterial resistance for several reasons, the foremost reason might be fungal agents were not recognized as important animal and human pathogens, until relatively in recent past. But the initial studies of antifungal drug resistance in the early 1980s, have accumulated a wealth of knowledge concerning the clinical, biochemical, and genetic aspects of this phenomenon. Presently, exploration of the molecular aspects for antifungal drug resistance has been undertaken. Recently, the focus was on several points like developing a more detailed understanding of the mechanisms of antimicrobial resistance, improved methods to detect resistance when it occurs, methods to prevent the emergence and spread of resistance and new antimicrobial options for the treatment of infections caused by resistant organisms.

Keywords: Antifungal, Incidence, Infection, Disease, Immunosuppression, Human, Population.
\end{abstract}

\section{Reasons for increase in the prevalence of fungal infections}

* $\quad$ Changes in Vetero-medical practices

* $\quad$ More widespread use of therapies that depress the animal's immune system

* The frequent and often indiscriminate use of broad-spectrum antibacterial agents

* The emerging or re-emerging mmunosuppressive diseases such as Chicken anemia virus infection, Bovine Viral Diarrhoea, Infectious Bursal Disease, AIDS etc. that may increase the chance of fungal infections.

\section{Commonly used antifungal drugs}

The most commonly used antifungal drugs are broadly classified into those of systemic and topical based on their route of administration.

* Systemic administration anti fungal drugs are Griseofulvin, Amphotericin B, Flucytosine and Azole compounds.

* Topical anti fungal agents are Amphotericin B, Tolnaftate, Clotrimazole, Miconazole, Ketoconazole and Nystatin.
Mechanism of action of antifungal drugs

* Three common classes of antifungal drugs

I. Ergosterol Biosynthesis Inhibitors

II. Polyenes

III. 5-Flucytosine (5-FC)

Properties of Antifungal agents

\begin{tabular}{|l|l|l|l|}
\hline $\begin{array}{l}\text { Action } \\
\text { target }\end{array}$ & Class & Type & $\begin{array}{l}\text { Antifungal } \\
\text { agent }\end{array}$ \\
\hline $\begin{array}{l}\text { Membrane } \\
\text { (Enzymatic } \\
\text { pathway) }\end{array}$ & $\begin{array}{l}\text { Ergosterol } \\
\text { Biosynthesis } \\
\text { Inhibitors }\end{array}$ & Allylamines & $\begin{array}{l}\text { Naftifine, } \\
\text { Terbinafine } \\
\text { Thicarbomates } \\
\text { Tolnaftate } \\
\text { Tolciclate } \\
\text { Ketoconazole } \\
\text { Miconazole } \\
\text { Fluconazole } \\
\text { Itraconazole } \\
\text { Voriconazole }\end{array}$ \\
\hline $\begin{array}{l}\text { Membrane } \\
\text { Composition }\end{array}$ & Polyenes & Azole based & $\begin{array}{l}\text { Amphotericin B } \\
\text { Nystatin }\end{array}$ \\
\hline $\begin{array}{l}\text { Protein } \\
\text { Synthesis }\end{array}$ & 5-flucytosin & --- & FCC \\
\hline (5FC) & & \\
\hline
\end{tabular}

Veterinary World Vol.2, No.5, May 2009 
I. Mechanism of action of Ergosterol biosynthesis inhibitors: Ergosterol is major sterol of the fungal plasma membrane, which is analogous to cholesterol in mammalian cells and is important as fungal membrane component for fluidity, asymmetry of the membrane, proper functioning of many membranebound enzymes like chitin synthetase, which is important for proper cell growth and division.

II Mechanism of action of Polyenes: Amphotericin B and nystatin, intercalate into membranes, forming a channel, through which cellular components, especially potassium ions leak, and there by destroy the proton gradient within the membrane

III Mechanism of action of 5-Flucytosine: 5-FC is taken up into the cell by a cytosine permease than with help of cytosine deaminase 5-FC deaminated into 5 -fluorouracil (FU). Cellular pyrimidine- processing enzymes convert 5-fluorouracil in to 5-fluoro-dUMP (FUMP) and 5-fluoro-dUTP (FUTP). FUMP which is a specific inhibitor of thymidylatesynthetase, an essential enzyme for DNA synthesis where as FUTP which is incorporated into RNA disrupting protein synthesis.

\section{Factors Contributing Antifungal Drug resistance}

Fungal factors: Species, Minimum Inhibitory Concentration (MIC), Cell types, Serotype, Genomic stability of strain and size of population.

Drug factors: Fungistatic nature of drug, Dosing, Frequency, Quantity, Cumulative dose, Pharmacokinetics.

Host Factors: Immune status, Site of infection, Severity of infection, Presence of foreign materials, Abscess formation, Patient noncompliance with drug regimen.

\section{Mechanism of Resistance}

\section{(A) Cellular mechanism of antifungal drug resistance}

i) Change to a more resistant species.

ii) Change to a more resistant strain.

iii) Genetic alterations that render a strain resistant.

iv) Transient gene expression that renders a cell temporarily resistant.

v) Alteration in cell type.

vi) Alteration in the fungal population.

(i) Change to a More Resistant Species

For each species, average MIC to particular drug can be determined. from MIC analysis, C. krusei is intrinsically more resistant to azole drugs than $C$. albicans. Intrinsic resistance of $C$. krusei and $C$. glabrata to azole drugs resulted in an increased frequency of resistant species in population. Azole drugs used, suppress the growth of sensitive $C$. albicans but allow the growth of more resistant strains or species. (ii) Change to More Resistant Strain: Azole sensitive and resistant isolates obtained from AIDS patients and analyzed by pulsed-field gel electrophoresis (molecular karyotyping) and restriction fragment length polymorphism (RFLP). The sensitive and resistant isolates differed by RFLP but not by karyotyping.

(iii) Genetic alterations that render a strain resistant: Strain resistant, exposure to the drug over long period of time and sensitive strain can become resistance. It is hypothesized that the drug itself does not cause resistance, and selects more resistant cells in the population. Genetic mutation occurs in all organisms at a low frequency. Under selective drug pressure specific random mutations that render the cell slightly more resistant dominant strain in the population.

(iv) Transient gene expression that renders a cell temporarily resistant: Candida cell can alter its phenotype, probably through transient gene expression, Candida cells become resistant in the presence of the drug, but the resistant phenotype can revert quickly to a susceptible phenotype once the drug pressure is eliminated.

(v) Alteration in Cell Type: C. albicans can be divided into two serotypes, $A$ and $B$, based on carbohydrate surface markers, serotype $B$ strains of $C$. albicans are more sensitive to azoles (specifically ketoconazole), but are more resistant to 5-FC than the A serotype strains (Odds, 1988).

(vi) Alterations in the fungal population: Variation within a population of strain for susceptible antifungal drug may be reflected in the form of antifungal drug resistance.

\section{(B) Molecular mechanisms of resistance}

(1) Azole Resistance

i. Reduction of the drug import

ii. Alterations in intracellular drug processing

iii. Alterations in the target enzyme

iv. Alterations in other enzymes in the ergosterol biosynthetic pathway

v. Alterations in efflux pumps

(i) Reduction of the drug import

Defect in drug import is first line of mechanism of drug resistance. Cells can alter the composition of the membrane to prevent drugs entering in to the cells. Change in sterol and phospholipids components of cytoplasmic membrane contribute to resistant of $C$. albicans.

(ii) Alterations in intracellular drug processing: Little analysis of drug modification or degradation within a resistant cell has been performed for the veteromedically important fungi. Azoles are inert to metabolism by $C$. albicans.

(iii) Alterations in the target enzyme: Common mechanism of drug resistance. For azole based 
antifungal drugs; it is the ergosterol biosynthetic pathway. The genes involved in the ergosterol biosynthetic pathway are ERG1, ERG2, ERG3, ERG4, ERG5, ERG6, ERG7, ERG11/ERG16, ERG24, ERG25, ERGX and ERGY (Bard et al., 1996).ERG1 to ERG12, ERG20, ERG24, ERG25 have been identified in C. albicans. Lanosterol demethylase is the product of ERG11 gene, a point mutation in ERG11 due to replacement of an arginine with lysine at amino acid 467 (R467K).

(iv) Alteration in other enzymes in the Ergosterol biosynthetic pathway: Another common mechanism of drug resistance is modification of the target enzyme and/or other enzymes in the same biochemical pathway, modifications in the ergosterol pathway are likely to generate resistance not only to the drug to which the cells are exposed but also to related drugs. Plasmid complementation of the ERG3 mutation in $S$. cerevisiae suggests that the ERG3 mutation alone can cause azole resistance.

Eukaryotic cells contain two types of efflux pumps that are known to contribute to drug resistance:

(I) Adenosine triphosphate (ATP) -binding cassette transporters (ABCT) family

(II) Major facilitators (MF) family

\section{I) $\quad \mathrm{ABCT}$ family}

The ABCT are composed of four protein domains: two membrane-spanning domains (MSD), each consisting of six or seven transmembrane-spanning segments, and two nucleotide binding domains (NBD). The NBD of ABCT bind ATP through an ABC that consists of several conserved peptide motifs, including two Walker domains, a Signature domain and a Center domain. The ATP that is bound to the $A B C$ is used as a source of energy for the $A B C T$, although the mechanism by which the ATP energy causes transport of the substrate molecule is unknown.

Candida drug resistant (CDR) gene is found to be associated with azole based drug resistance. Level of CDR were higher in clinical azole resistance isolates than those in clinical susceptible ones, a deletion of CDR results in azole hyper susceptibility to azole drugs

The MF does not contain NBD. They are composed primarily of 12 to 14 transmembrane segments $(110,140)$. The MF use the proton motive force of the membrane (gradient of H1across the membrane) as a source of energy. MDR is the only MF gene reported having correlation with drug resistance 2) Non Azole resistance

i) Allylamine and thiocarbamate resistance: Resistance to Allylamine and thiocarbamates has not been reported, although resistant strains have been described for S. cerevisiae. Prolonged use will probably select for strain replacement with a resistant isolate or the development of secondary resistance in the original sensitive isolate (Anaissie et al., 1989).

(ii) 5-Flucytosine resistance: Primary or intrinsic resistance to $5-F C$ - common phenomenon $10 \%$ clinical isolates of $C$. albicans are intrinsically resistant and that of $30 \%$ will develop secondary resistance (Anaissie et al., 1989). Cryptococcus neoformans mutations are the most likely cause of 5-FC resistance in these yeasts, analysis of two genetic loci, FCY1 and FCY2 that can be mutated to produce 5-FC resistance.

Primary or intrinsic resistance to $5-\mathrm{FC}$ is usually the result of a defect in cytosine deaminase and secondary resistance to 5-FC in C. albicans is due primarily to a decrease in the activity of the uracil phosphoribosyl transferase (UPRTase), which is involved in the synthesis of FUMP and FdUMP. Of the two serotypes of $C$. albicans, serotype $B$ is more frequently associated with 5-FC resistance.

\section{Incidence of Antifungal resistance in Veterinary and Medical field}

There are few reported incidences on antifungal resistance both in Veterinary and medical field. Some of them are highlighted here. Jadhav, (2001) reported antifungal drug resistance exhibited by $C$. albicans isolated from man, animals and birds. From man (293), farm animals (97), and poultry (60) the prevalence rate of $C$. albicans was 11.02 per cent, 8.02 per cent, and 6.66 per cent, respectively. The antifungal drug resistance pattern was fluconazole 52 per cent nystatin 41 per cent clotrimazole 37 per cent amphotericin B 19 per cent, respectively.

Similarly, Thomas, (2004) reported antifungal drug resistance from Cryptococcus neoformans isolated from man, animals birds and environment. From man (120), animals (97), avian (96) and environment sources (127) the prevalence rate of Cryptococcus neoformans was 3.2\%, 3.09\%, 5.21\% and $9.09 \%$, respectively. Resistance to Clotrimazole and Fluconazole was 25.0 per cent, and that of Nystatin and Amphotericin-B was 50.0 per cent and 58.33 per cent, respectively.

Among the fungal isolates from canine cystitis, 59.0 per cent $C$. albicans and 42.0 per cent $C$. tropicalis were resistant to fluconazole; 62.0 per cent $C$. albicans and 43.0 per cent $C$. tropicalis were resistant to Amphotericin B.

Due to drug-resistant fungi, oropharyngeal Candidasis is a major problem for patients infected with Human immunodeficiency virus. Alteration in immune status associated with the AIDS epidemic, cancer chemotherapy, organ and bone marrow transplantation may be responsible to antifungal drugs 
resistance (White et al., 1998). Drug resistant strains of Candida albicans flared up in oral cavities during late stages of AIDS patients after antifungal drug therapy.

\section{Prevention and control of antifungal resistance}

Strategies to avoid and suppress the emergence of antifungal resistance have not been defined, approaches recommended for antibacterial resistance could be suggested, these includes,

(i) Prudent use of antifungal drugs

(ii) FAppropriate dosing with special emphasis on avoiding treatment with low antifungal dosage

(iii) Therapy with combinations of existing drugs

(iv) Treatment with the appropriate antifungal drugs

(v) Use of surveillance studies to determine the true

\section{Conclusions} frequency of antifungal drug resistance

There was no predominant mechanism contributing to resistance of antifungal drugs. Unlike resistance to antibiotics, antifungal drug resistance due to plasmid mediated mechanism has not been reported. With increased usage of different antifungal drugs, we may expect an increasing number of clinical fungal pathogens becoming resistance to these drugs. Cellular and molecular factors contribute to anti fungal drug resistance. Rapid identification of fungal pathogens and the measurement of the MIC of clinical isolates in vitro will be helpful.

Future Prospects: The mechanism by which the different factors that contribute to overall antifungal drug resistance is still in infancy stage. There is a need for considering several variables to minimize the risk for development of resistance. There is a clear need for the next generation of antifungal agents. The ongoing developments in the understanding of resistance will assist in the development of diagnostic strategies to identify resistant clinical isolates. Commonly used antifungal drugs are ergosterol biosynthesis inhibitors, polyenes, and 5-flucytosine (5-FC). In a resistant cell, the azoles are blocked from interacting normally with the target enzyme because the enzyme can be modified and/or overexpressed. With increased usage of different antifungal drugs, we may expect an increasing number of clinical fungal pathogens becoming resistant to these agents (Cohen, 1992).

\section{References}

1. Anaissie, E. R.; Hachem, C. K. and Bodey, G. P. (2003): Experimental hematogenous candidiasis caused by Candida krusei and Candida albicans: species differences in pathogenicity. Infect. Immun. 61: 12681271.

2. Ghannoum, M. A. and Louis, B. R. (1999): Antifungal agents: mode of action, mechanisms of resistance, and correlation of these mechanisms with bacterial resistance Clinical Microbiology Reviews. 12:501-517.

3. Jadhav, V. J. (2001): Human and animal infections caused by Candida albicans. M.V.Sc. thesis submitted to Gujarat Agricultural University, Anand.

4. Odds, F. C. (1988): Candida and candidiosis: a review and bibliography bailliere tindall, London, UK.

5. Thomas, L. (2004): Prevalence of Cryptococcus neoformans in man, animal and environment. M.V.Sc. thesis submitted to Anand Agricultural University, Anand.

6. White, T. C.; Marr, K. A. and Bowden, R. A. (1997): Cellular and molecular factors that contribute to antifungal drug resistance. Clinical Microbiology Reviews. 11: 382-402.

\footnotetext{
$* * * * * * * *$
} 